\title{
Sex-specific changes in autosomal methylation rate in ageing common terns
}

\author{
Britta Meyer ${ }^{1}$, Maria Moiron ${ }^{2}$, Calvinna Caswara ${ }^{3}$, William Chow ${ }^{4}$, Olivier Fedrigo ${ }^{5}$, \\ Giulio Formenti ${ }^{5}$, Bettina Haase ${ }^{5}$, Kerstin Howe ${ }^{4}$, Jacquelyn Mountcastle ${ }^{5}$, Marcela \\ Uliano-Silva $^{4}$, Jonathan Wood ${ }^{4}$, Erich Jarvis ${ }^{5}$, Miriam Liedvogel ${ }^{1}$, and Sandra Bouwhuis ${ }^{2}$ \\ ${ }^{1}$ Max Planck Institute for Evolutionary Biology \\ ${ }^{2}$ Institute of Avian Research \\ ${ }^{3}$ Berlin Center for Genomics in Biodiversity Research \\ ${ }^{4}$ Wellcome Sanger Institute \\ ${ }^{5}$ The Rockefeller University
}

February 26, 2022

\begin{abstract}
Senescence, an age-related decline in survival and/or reproductive performance, occurs in species across the tree of life. Molecular mechanisms underlying this within-individual phenomenon are still largely unknown, but DNA methylation changes with age are among the candidates. Using a longitudinal approach, we investigated age-specific changes in autosomal methylation of common terns, relatively long-lived migratory seabirds known to show senescence. We collected blood at 1-, 3- and/or 4-year intervals, extracted DNA from the erythrocytes and estimated autosomal DNA methylation by mapping Reduced Representative Bisulfite Sequencing reads to a new reference genome. We found autosomal methylation levels to decrease with age within females, but not males, and no evidence for selective (dis)appearance of birds of either sex in relation to their methylation level. Moreover, although we found positions in the genome to consistently differ in their methylation levels, individuals did not show such strong consistent differences. These results pave the way for studies at the level of genome features or specific positions, which should elucidate the functional consequences of the patterns we observe, and how they translate to the ageing phenotype.
\end{abstract}

\section{Sex-specific changes in autosomal methylation rate in ageing common terns}

Britta S. Meyer ${ }^{1}$, Maria Moiron ${ }^{2}$, Calvinna Caswara ${ }^{3}$, William Chow $^{4}$, Olivier Fedrigo ${ }^{5}$, Giulio Formenti ${ }^{5}$, Bettina Haase $^{5}$, Kerstin Howe ${ }^{4}$, Jacquelyn Mountcastle ${ }^{5}$, Marcela Uliano-Silva ${ }^{3,4 \S, 6}$, Jonathan Wood ${ }^{4}$, Erich D. Jarvis ${ }^{5,7}$, Miriam Liedvogel ${ }^{* 1,2 \S}$, Sandra Bouwhuis*2

*equal contributions

$\S$ current address

${ }^{1}$ Max Planck Institute for Evolutionary Biology, Behavioural Genomics; August-Thienemann-Str. 2; 24306 Plön; Germany

${ }^{2}$ Institute of Avian Research; An der Vogelwarte 21; 26386 Wilhelmshaven; Germany

${ }^{3}$ Berlin Center for Genomics in Biodiversity; Königin-Luise-Straße 2-4 Gartenhaus; 14195 Berlin Germany

${ }^{4}$ Wellcome Sanger Institute, Wellcome Genome Campus, Cambridge CB10 1SA, UK

${ }^{5}$ The Rockefeller University, 1230 York Avenue, New York, NY 10065, USA 
${ }^{6}$ Department of Evolutionary Genetics, Leibniz Institute for Zoo and Wildlife Research (IZW)

${ }^{7}$ Howard Hughes Medical Institute, 4000 Jones Bridge Rd, Chevy Chase, MD 20815

Corresponding author: bmeyer@evolbio.mpg.de

\begin{abstract}
Senescence, an age-related decline in survival and/or reproductive performance, occurs in species across the tree of life. Molecular mechanisms underlying this within-individual phenomenon are still largely unknown, but DNA methylation changes with age are among the candidates. Using a longitudinal approach, we investigated age-specific changes in autosomal methylation of common terns, relatively long-lived migratory seabirds known to show senescence. We collected blood at 1-, 3- and/or 4-year intervals, extracted DNA from the erythrocytes and estimated autosomal DNA methylation by mapping Reduced Representative Bisulfite Sequencing reads to a new reference genome. We found autosomal methylation levels to decrease with age within females, but not males, and no evidence for selective (dis)appearance of birds of either sex in relation to their methylation level. Moreover, although we found positions in the genome to consistently differ in their methylation levels, individuals did not show such strong consistent differences. These results pave the way for studies at the level of genome features or specific positions, which should elucidate the functional consequences of the patterns we observe, and how they translate to the ageing phenotype.
\end{abstract}

Keywords: aging, avian senescence, epigenetics, ontogeny, RRBS,

Sterna hirundo

\title{
Introduction
}

Senescence is a within-individual decline in survival probability (actuarial senescence) and/or reproductive performance (reproductive senescence) with age. Although the rate and shape of the decline vary both among and within species, this detrimental process occurs in species across the tree of life (Shefferson et al. 2017). It is hypothesized to have evolved because unavoidable extrinsic mortality reduces the strength of selection against poor performance with age (Fisher 1930; Medawar 1952; Williams 1957; Hamilton 1966). From a genetic point of view, this so-called 'selective shadow' could allow for the accumulation of lateacting deleterious mutations over evolutionary time (mutation accumulation, Medawar 1952), or for selection favouring alleles with beneficial effects early, but detrimental effects late in life (antagonistic pleiotropy, Williams 1957). Moreover, early-life investment of limited resources in reproduction over that in perfect somatic maintenance and repair would also be evolutionarily beneficial, such that senescence could be a 'best-of-a-bad-job' consequence of accumulated, unrepaired damage (disposable soma, Kirkwood 1977).

Although the classical theories of mutation accumulation and antagonistic pleiotropy assume a purely sequence-level genetic basis to senescence, the disposable soma theory does not. Given its firm foundation in life history theory, resource acquisition and allocation (sensu van Noordwijk \& de Jong 1986) are important facets to consider. Both are known to show phenotypic plasticity in response to environmental variation (e.g.

Erikstad et al. 1998; Descamps et al. 2016), and experimental manipulation of one of these facets without adjustment of the other is known to affect rates of senescence (e.g. Boonekampet al. 2014). As such, part of the senescence process is also expected to be underpinned by more flexible, regulatory processes (Wilson et al. 2008). In line with this, numerous studies across taxa have shown that senescence is underpinned by both genetic and epigenetic processes (Sen et al. 2016; Melzer et al.2020).

Epigenetic processes are those that affect the regulation of gene expression (Holliday 2006). This regulation is complex and encompasses several mechanisms, a major one of which is DNA methylation, the addition of a methyl-group to the fifth carbon site of a cytosine in a $\mathrm{CpG}$ (5'-C-phosphate-G-3') dinucleotide context (Jaenisch \& Bird 2003; Miranda \& Jones 2007; Suzuki \& Bird 2008). Age-specific DNA methylation has been described for many model species (e.g. Maegawaet al. 2010; Hannum et al. 2013; Tharakan et al.2020), and 
the methylation status of specific CpGs has been shown to be a powerful predictor of both chronological and biological age, i.e. to function as an epigenetic clock (Bocklandt et al. 2011). Using a cross-sectional analytical approach, epigenetic clocks have meanwhile been characterized for a variety of organisms, including humans (Weidner et al. 2014), dogs and wolves (Thompson et al. 2017) and cetaceans (Robeck et al. 2021). Importantly and additionally, a rare longitudinal study following the same set of human twins across 10 years, revealed (i) the methylation of CpG sites to change with agewithin subjects, (ii) this change to mostly occur in gene-sets involved in ageing-related degenerative disorders, and (iii) most of the change to be explained by individual-specific environmental factors rather than inherited (genetic) factors (Tan et al. 2016). Although age-specific methylation seems the norm at least in model organisms, the direction of methylation changes with age is harder to predict: both passive non-directional changes (representing epigenetic drift, Fraga et al. 2005; Tan et al. 2016), and active directional changes (hyper- and hypomethylation, Zampieri et al.2015; Ciccarone et al. 2018; but see Unnikrishnan et al.2019) have been reported.

Research on senescence, including its epigenetic underpinning, has so far mostly focused on humans or model organisms kept under controlled laboratory conditions. Extending the taxonomic range and incorporating field studies is crucial to understand the evolutionary ecology of senescence (Monaghan et al. 2008). For this extension, birds are an especially interesting taxon. They have longer life spans relative to their body size than mammals (e.g. Lindstedt \& Calder 1976), and various populations of birds with vastly different life histories have been studied over several decades, such that many basic insights into their senescence patterns can now be obtained (Bouwhuis \& Vedder 2017). Bird genomes are rather compact, show high levels of synteny across species (Zhang et al. 2014), and, similar to those of other vertebrates, are globally methylated (Suzuki \& Bird 2008; Li et al. 2011, Sepers et al. 2019). With respect to age-specific DNA methylation in birds, we are aware of the existence of an epigenetic clock for short-tailed shearwaters (Ardenna tenuirostris ; De Paoli Iseppi et al. 2019), and of findings of early-life within-individual increases in global methylation in both great tit (Parus major ; Watson et al. 2019) and zebra finch (Taeniopygia guttata; Sheldon et al. 2020) nestlings. However, we are not aware of any study reporting age-specific DNA methylation patterns in avian adulthood, or in avian late-life specifically.

When studying age-specific trait expression, many studies, including most of those producing epigenetic clocks or otherwise studying age-specific differences in DNA methylation patterns, use cross-sectional samples and analysis tools. Patterns revealed by cross-sectional approaches, however, are the result of a combination of within- and among-individual processes. If we aim for understanding the within-individual process of senescence, we need to account for the effect of compositional changes of a population, for example when birds with a certain level of DNA methylation are more likely to die and selectively disappear from the study population (e.g. Vaupel \& Yashin 1985; Forslund \& Part 1995). Mixed-effect models applied to (partly) longitudinal data to specifically test whether within-individual patterns and population-level patterns are the same, or differ, are a powerful analytical tool to do so (van de Pol \& Wright 2009). Across taxa, efforts have increasingly been made to validate and complement findings from cross-sectional analyses of senescence through longitudinal studies (Nussey et al. 2008; Gaillard et al.2017), and this methodological turn is also reflected in studies of human DNA methylation (Bollati et al. 2009; Tan et al.2016). Longitudinal studies of DNA methylation and ageing in model species and natural populations are, however, missing and needed (Bellet al. 2019).

Here, we report on a longitudinal study of autosomal methylation levels in the common tern. Common terns are long-lived migratory seabirds whose patterns of senescence have been the topic of various studies. Although breeders of both sexes show little sign of reproductive senescence - they breed earlier in the year and fledge more offspring as they grow older (Nisbet et al. 2002; Zhang et al. 2015c; Nisbetet al. 2020) breeding and survival probabilities are known to decline with age (Zhang et al. 2015b; Vedder et al.2021b). In addition, there is evidence for sex-specific transgenerational senescence, with daughters of older mothers and sons of older fathers suffering from reduced lifetime reproductive success (Bouwhuis et al. 2015). Studies aiming to identify a molecular basis for these within- and transgenerational effects have so far focused on telomere dynamics, and found that: (i) telomeres shorten with age (Bichet et al. 2020); (ii) telomere length is genetically correlated with lifespan (Vedder et al. 2021a); and (iii) paternal age is negatively correlated 
with offspring telomere length (Bouwhuis et al. 2018). The explanatory power of telomere length, however, is very low for all of these patterns (e.g. 1.1\% of phenotypic variation in lifespan is explained by additive genetic variation in telomere length, Vedder et al. 2021a), such that additional mechanisms are expected. To evaluate whether age-specific changes in global DNA methylation could be such a mechanism, we sequenced and de novo assembled a chromosome-scale high-quality reference genome and used it to compare withinindividual age-specific changes in DNA methylation at shared sites across the genome. Although rates of within-generational senescence do not differ between the sexes (Zhanget al. 2015a), transgenerational effects are known to be sex-specific (Bouwhuis et al. 2015), such that we also considered sex-specificity of any patterns in autosomal methylation status.

\section{Materials and Methods}

\section{Study population}

The data we present were collected as part of a long-term individual-based study of a mono-specific common tern colony located on six artificial concrete islands at the Banter See ( $\left.53^{\circ} 36^{\prime} \mathrm{N}, 08 \mathrm{deg} 06^{\prime} \mathrm{E}\right)$ in Wilhelmshaven, Germany. Fledglings from this colony have been marked with metal rings since 1984, and subcutaneously injected with transponders since 1992 (Becker \& Wendeln 1997). This transponder-marking combined with placement of antennae on elevated platforms on the edges of the colony site and around each nest during incubation (shared between both parents), allows for a well-described family structure of all philopatric birds (e.g. Moiron et al.2020).

\section{Reference genome}

To create the chromosome-scale reference genome, blood of one adult female common tern was collected in $100 \% \mathrm{EtOH}$ and stored at -80degC. Four sequence datasets were generated following the VGP 1.5 pipeline (Rhieet al. 2021): 67.91x Pacific Biosciences (Pacbio) continuous long reads (CLR); 698.35x Bionano Genomics optical maps; 169.30x 10X Genomics linked-reads; and 79.62x Arima Hi-C Illumina reads.

Briefly, 30ug of High Molecular Weight DNA (HMW DNA) was isolated from the whole blood sample using a modified (for avian nucleated erythrocytes) agarose plug protocol of the Bionano Prep Blood and Cell Culture DNA Isolation Kit (cat no. RE-130-10). Lysates were embedded into agarose plugs, followed by Proteinase K and RNase A treatments and 1X TE drop dialysis purification. To create the Pacbio data, DNA was sheared using a 26G blunt end needle (Pacbio protocol PN 101-181-000 Version 05) to approximately $\sim 40 \mathrm{~kb}$ fragment length. We used $10 \mu \mathrm{g}$ of this fragmented DNA to generate a large-insert Pacbio library using the Pacific Biosciences Express Template Prep Kit v1.0 (\#101-357-000). The library was then size selected (>15kb) using the BluePippin system (Sage Science). The resulting PacBio Library was sequenced on 10 PacBio 1M v3 smrtcells (\#101-531-000) on a Sequel instrument with the sequencing kit 3.0 (\#101427-500) and a 10 hours movie with 2 hours pre-extension time. Unfragmented HMW DNA was used to generate a linked-read library on the 10X Genomics Chromium (Genome Library Kit \& Gel Bead Kit v2 PN-120258, Genome Chip Kit v2 PN-120257, i7 Multiplex Kit PN-120262). We sequenced this 10X library on an Illumina Novaseq S4 150bp PE lane. uHMW DNA was labeled for Bionano Genomics optical mapping using the Bionano Prep Direct Label and Stain (DLS) Protocol (30206E) and 1 flow cell was run on the Saphyr instrument. Hi-C libraries were generated with the Arima Genomics v1.0 2-enzyme protocol (P/N: A510008), according to the manufacturer's protocol and sequenced on Illumina HiSeq X.

The resulting four data types were processed using the VGP v1.5 pipeline (Rhie et al. 2021), which includes: assembling Pacbio contigs using FALCON v2018.31.08-03.06 ; FALCON-Unzip v6.0.0.47841; purging false haplotype duplications with purge_haplotigs v1.0.3+ 1.Nov. 2018 ; scaffolding with $10 \mathrm{X}$ with scaff10x v4.1.0; scaffolding with Bionano Solve DLS v3.2.1 ; scaffolding with Hi-C data with Salsa HiC v2.2 ; filling in gaps and polishing for base call accuracy with CLR and Arrow smrtanalysis v6.0.0.47841 ; and polishing with Illumina short reads with longranger align v2.2.2 ; and freebayes v1.3.1 . The resulting assembly was then manually curated to fix any errors, using $g E V A L$ and Hi-C short read linked-read mapping profiles as described in Howe et al. (2021).BUSCO v4.1.4 with the bird lineage dataset (aves_odb10) was used to assess 
assembly completeness. The reference genome was submitted to NCBI with the following accession number: GCA_009819605.1, as part of the Vertebrate Genome Project (VGP) (https://vgp.github.io/genomeark). Our reference genome has GC-rich promoter regions due to the use of Pacbio long reads that get through them (Rhie et al. 2021; Kim et al. 2021). Reference genomes consisting of short-read assemblies (e.g., using Illumina reads) exhibit GC bias, where GC-rich regions such as promoters could be incorrectly assembled or even missing (Kim et al. 2021).

\section{Methylation sequencing}

Blood of 34 adults of 17 breeding pairs was sampled in the years $2013(\mathrm{~N}=16), 2014(\mathrm{~N}=32)$ and 2017 $(\mathrm{N}=26)$ using larval stages of the bloodsucking bug Dipetalogaster maximus. Bugs were placed into dummy eggs with holes through which they could stick out their mouth pieces and placed in the nests of incubating focal birds (Beckeret al. 2006; Arnold et al. 2008; Bichet et al.2019). After 20-30 minutes, "bug eggs" were collected and the focal birds' blood, sucked by the bug, was removed from the bugs' abdomen using a syringe. Upon collection, whole blood was stored in EDTA buffer $(2 \%)$ in a fridge $\left(3-7^{\circ} \mathrm{C}\right)$ for up to 3 weeks, before red blood cells were transferred to glycerol buffer (40\%) and frozen at $-80^{\circ} \mathrm{C}$. Each bug was used only once to prevent contamination of blood samples.

Genomic DNA was extracted and libraries for RRBS were generated as described in Klughammer et al. (2015), following standard steps such as MspI digestion, end-fill-in, A-tailing and size selection. This included the enrichment of the libraries with Pfu-Turbo Cx Hotstart DNA polymerase to allow the assessment of the bisulfite conversion rate. After clean-up and quality control, libraries were sequenced with an Illumina HiSeq 4000 (50bp SE).

\section{Methylation data selection}

Across all 74 samples, individuals were sequenced with an average of 51,482,011 (range: 24,942,071 $82,760,425)$ reads. After conversion of unmapped bam files to fastq with SamToFastq.jar frompicard v1.118 , the quality of the sequencing reads was checked using Fastqc v0.11.5 (Andrews 2010) and Multiqc v1.8 (Ewels et al. 2016). Spiked-in sequences were used to estimate over- and underconversion using RefFreeDMA (Klughammeret al. 2015). Trimgalore v0.3.3 (implemented inRefFreeDMA; Krueger et al., 2021) was used to remove adaptors to guarantee good mapping and remove low quality bases ([?]20) as well as short read fragments ([?] $16 \mathrm{bp})$.

We used Bismark v0.22.3 (Krueger \& Andrews 2011) to prepare and index the reference genome for subsequent mapping of the bisulfite-converted reads using Bowtie 2 v2.4.1 (Langmead \& Salzberg 2012), allowing for one mismatch (-score_min L,0,-0.20). Methylation extraction was conducted with Bismark Extractor v0.22.3 (Krueger \& Andrews 2011) with the 'ignore' option to remove unmethylated cytosines introduced during the end-repair step. The R-package Methylkit v.1.16.1 in R v.4.0 (Akalin et al. 2012; R Core Team, 2021) and its function methRead were used to load and analyse the methylation calls.

Methylation calls were filtered to those with a minimum coverage of 10 reads per CpG position. CpGs with a coverage $>99.9$ th percentile most likely result from PCR bias and were removed. Across all 74 samples, there were on average 6,361,176 $\mathrm{CpG}$ positions before, and 539,538 $\mathrm{CpG}$ positions after, filtering for $>10 x$ coverage. Methylation call distributions between samples were normalized using thenormalizeCoverage function implemented in Methylkit to reduce the bias of systematic oversampling in some samples. We then merged $\mathrm{CpG}$ positions of the different samples using the unitefunction to make sure positions were sequenced in at least $70 \%(\mathrm{~N}=52)$ of the samples to facilitate a longitudinal analysis approach. This also meant we only covered $\mathrm{CpG}$ positions on autosomes, as CpGs from the sex chromosomes were excluded. Following Meng et al. (2010) and Sziraki et al. (2018), sites that showed little or no variation were removed as well, applying a threshold of a standard deviation $<0.1$. This resulted in 1,365,573 observations of 23,647 sites, used to determine methylation levels as the fractional methylation rate per CpG position per individual.

\section{Statistical analyses}

To identify and partition sources of variation in autosomal methylation levels, we used the $\mathrm{R}$ package 
glmmTMB (Brooks et al. 2017) to run a generalized linear mixed model (GLMM) with the BFGS algorithm as an optimizer, using the number of methylated and unmethylated Cs (represented by the sequenced Cs and Ts) for each position as our dependent variable, assuming a binomial error distribution (Lea et al. 2017). As fixed effects we added sex (as a two-level class variable using males as the reference category) and age (as a covariate). With respect to the latter, each individual's age was partitioned into an 'average age' and 'delta age' component following van de Pol \& Wright (2009). Average age was calculated as the average of all ages at which we assessed a bird's autosomal methylation level, while delta age was calculated as the difference between the bird's actual age and its average age (i.e. delta age $=$ age - average age). When adding both age variables as covariates, average age represents the among-individual, and delta age the within-individual age effect (van de Pol \& Wright 2009). If the among- and within-individual age effects were to differ, this would indicate that the effect of age among individuals cannot be explained by changes within individuals, revealing age-specific selective (dis)appearance of individuals with certain levels of methylation (van de Pol \& Wright 2009). We additionally included the interaction between sex and delta age in our model to test for sex-differences in the within-individual age trajectory of methylation. Random effects included were 'bird identity', genomic position ('position identity') and an observation-level random effect ('observation'). The latter was added to account for overdispersion, which was detected using the $\mathrm{R}$ package performance (Ludeckeet al. 2021), whereas the first two were included to account for pseudoreplication caused by repeated sampling of individuals and genomic positions, respectively. Model evaluation and summary of parameter estimates and statistics were conducted using the $\mathrm{R}$ packageparameters (Ludecke et al. 2020). The marginal effects of the interaction were plotted using the R-package sjplot test (Ludecke 2018).

\section{Results}

\section{Reference genome}

The reference genome was generated using 68x PacBio sequencing reads, Bionano Genomics optical maps, 10X Genomics linked-reads and Arima Hi-C reads. This allowed us to scaffold the assembly to chromosomelevel (Rhie et al. 2021) and we successfully assigned $99.3 \%$ of the assembled sequence to 25 identified autosomes, two sex chromosomes and the mitochondrial genome, leaving only 95 scaffolds unlocalised. The total length of the primary haplotype assembly was $1.23 \mathrm{Gbp}$ with a contig N50 of $22.0 \mathrm{Mb}$ and a scaffold $\mathrm{N} 50$ of $85.5 \mathrm{Mb}$. It included $96 \%$ complete assembled single copy genes according to BUSCO analysis, with only $1.3 \%$ fragmented, $0.4 \%$ falsely duplicated, and $2.3 \%$ missing $(\mathrm{n}=8,338$ genes $)$. This represents a high-quality assembly, surpassing the aspired VGP contiguity metrics $\sim 20$-fold (Rhieet al 2021).

\section{Age-specific global methylation}

Across the 74 samples, 29,044,662 RRBS reads ( $56 \%$ mapping efficiency) could be uniquely mapped to the reference genome. The mean bisulfite conversion rate was $99.2 \%$. When analyzing sources of variation in methylation rates across the $23,647 \mathrm{CpG}$ positions occurring in at least $70 \%$ of samples, we found a significant interaction between sex and delta age (Table 1). This interaction showed that methylation rates declined with age within individual females, but did not change with age within individual males (Figure 1). The effect of average age was non-significant and the credible intervals of the average and delta age components strongly overlapped (Table 1), suggesting no selective (dis)appearance of individuals based on their autosomal methylation rates. When assessing the random effects, most variance was explained by position identity (Table 1), showing that genomic positions differ in their average level of methylation. Bird identity explained variation in methylation rates to a much lesser extent (Table 1), such that there is little evidence for consistent differences in methylation among individuals.

\section{Discussion}

DNA methylation patterns at CpG sites are increasingly used as biomarkers, so-called epigenetic clocks, to predict both chronological and biological age across species and taxa (e.g. Lu et al. 2021). How DNA methylation changes within individuals and whether it can explain phenotypic senescence patterns, however, is still largely unknown (Bell et al. 2019). Here, we used blood samples from common terns collected at 1-, 3- 
and/or 4-year intervals and a longitudinal analysis approach to investigate whether autosomal methylation levels changed with age within individual birds, and whether any change differed between males and females sharing environments and broods. We found female genomes to become less methylated as these females aged, whereas there was no such age-specific decline of autosomal methylation in males. Moreover, we found the estimates for the within- and among-individual components of age to be similar, such that there was no indication for selective (dis)appearance of individuals based on their methylation pattern. Finally, we provide evidence for positions in the genome to consistently differ in their methylation levels, whereas evidence for consistent differences among individuals was considerably less.

Our finding of female common terns showing a decrease in methylation as they aged fits with findings of global hypomethylation in older compared to younger mammals (Zampieri et al. 2015; Ciccarone et al.2018; Sziraki et al. 2018; but see Unnikrishnan et al. 2019). Such methylation loss is thought to partly originate from demethylation of large regions of repetitive sequences, CpG-poor promoters or large hypomethylated blocks of "open sea" regions outside the CpG islands (Bollati et al. 2009; Heyn et al. 2012; Yuan et al.2015). Whether these changes affect chromatin configuration and thus genome (in)stability, and whether changes in promoter methylation interact with histone modifications and transcription factors to alter expression remains to be investigated (Zampieri et al. 2015; Ciccarone et al. 2018).

Male terns, in contrast, showed no signs of decreased methylation as they grew older. Although many studies developing epigenetic clocks have assumed age-related changes to be similar across the sexes and used mixedsex datasets to obtain them (e.g. Raj et al. 2021; Horvathet al. 2021), others have found sex-differences in these clocks (e.g. in some human ethnicities (Horvath et al. 2016), baboons (Anderson et al. 2021) or elephants (Prado et al. 2021)). Moreover, a rare longitudinal study in a wild population of roe deer also showed sex-specific epigenetic clock regions, with an accelerated ageing signal in males, which are known to undergo stronger survival senescence in this species (Lemaitre et al. 2021). Combined with our findings, this suggests that methylation tests for sex-specificity should best be the norm.

Interestingly, male and female terns from our study population do not differ in the onset or rate of senescence in survival or breeding probabilities (Zhang et al. 2015b; Vedder et al. 2021b), such that sex-specificity in the ageing process is only found in how parental age affects the quality of the offspring that recruit back into the population (with maternal age negatively affecting the reproductive performance of daughters and paternal age negatively affecting survival of sons (Bouwhuis et al. 2015)). As such, we did not necessarily expect sex differences in the age-specificity of the birds' autosomal methylation level. The fact that we were able to observe them, raises the question of which site-specific methylation patterns drive the pattern observed on the global level. As mentioned above, global loss is thought to originate from the demethylation of specific regions: repetitive sequences, CpG-poor promoters or large hypomethylated blocks of "open sea" regions outside the CpG islands (Bollati et al.2009; Heyn et al. 2012; Yuan et al. 2015). CpG island promoters, on the other hand, have been found to show age-specific increases in methylation (Heyn et al. 2012; Day et al.2013). As such, global demethylation may perhaps be compensated for by such increases in males, but not females. In combination with the fact that we found genomic positions to consistently differ in methylation levels, this stresses the need for moving from the level of global autosomal methylation assessment that we and others (e.g. Watsonet al. 2019; Sheldon et al. 2020) have started with, to fully annotating the (common tern) genome and studying patterns across genomic features and focal sites.

Besides finding a sex-specific within-individual change in autosomal methylation level with age, and significant among-position consistency in methylation level, we found little evidence for consistent amongindividual levels of autosomal methylation across years, or for selective (dis)appearance of birds in relation to their methylation level. This suggests that birds differentially change their autosomal methylation from year to year, with these changes perhaps reflecting their individual-specific condition or environment, but not relating to their recruitment as a breeder in the study population or their local survival. Implementing a random regression analytical framework is data-hungry and not possible with our current dataset, but linking among-year changes in e.g. body mass or other measures of physiology seems a promising research avenue, especially when taking the analyses to a site-specific level, such that distinct genotype-methylation-phenotype 
correlations can be identified.

The strength of our study lies in its longitudinal sampling and analytical approach, which allows us to characterise within-individual changes, rather than infer them from cross-sectional data. At the same time, however, this sampling approach may come with some limitations. Non-destructive, longitudinal sampling in natural populations often relies on using blood as the focal tissue, but how DNA methylation of (in our case) erythrocytes translates to phenotypes mostly remains an open question that needs addressing (Husby 2020), ideally in experimental study systems. Moreover, because we used RRBS, a high-throughput and low-cost method, to assess methylation, it is important to realise that this method introduces biases towards high density CpG regions (Smith et al. 2009; Gu et al. 2011); we have focally answered the question of what happens at CpG islands during ageing (Beck et al. 2021). Keeping this in mind, however, our study has provided evidence for a sex-specific within-individual change in autosomal methylation with age and shown that different positions in the genome are consistently differentially methylated, such that future work, changeing the perspective from genome-wide average estimates to the specific genome feature or base pair level, can elucidate whether, where and how much methylation might affect ageing males and females, as such establishing a longitudinal epigenetic clock.

\section{Acknowledgements}

We are indebted to Peter H. Becker for setting up the long-term individual-based common tern study at the Banter See and to Gotz Wagenknecht and the many scientists, students and field assistants who collected the data across the years. We express our gratitude to Johanna Klughammer, Amelie Nemc and Christoph Bock for their advice on sequencing strategy and for performing the RRBS sequencing. We also thank Michaela Schwarz for help with DNA extraction, Kristian Ullrich for his support in solving bioinformatics hurdles, and Melanie J. Heckwolf and our epigenetic journal club for inspiring discussions on DNA methylation. The study was performed under licenses of the city of Wilhelmshaven and the Lower Saxony State Office for Consumer Protection and Food Safety, Germany. This study was supported by the Max Planck Society through a MPRG grant (MFFALIMN0001 to ML), a sequencing grant (PSLIMN6000 to ML and SB), and the "Norddeutscher Wissenschaftspreis 2018" (BSM, SB and ML).

\section{References}

Akalin A, Kormaksson M, Li S et al. (2012) methylKit: a comprehensive R package for the analysis of genome-wide DNA methylation profiles. Genome biology, 13, R87.

Andrews S (2010) A quality control tool for high throughput sequence data. bioinformatics.babraham.ac.uk

Anderson JA, Johnston RA, Lea AJ et al. (2021) High social status males experience accelerated epigenetic aging in wild baboons.eLife, 10 .

Arnold JM, Oswald SA, Voigt CC et al. (2008) Taking the stress out of blood collection: comparison of field blood-sampling techniques for analysis of baseline corticosterone. Journal of Avian Biology , 39 , 588-592.

Beck D, Ben Maamar M, Skinner MK (2021) Genome-wide CpG density and DNA methylation analysis method (MeDIP, RRBS, and WGBS) comparisons.Epigenetics .

Becker PH, Wendeln H (1997) A New Application for Transponders in Population Ecology of the Common Tern. $99,534-538$.

Becker PH, Voigt CC, Arnold JM, Nagel R (2006) A non-invasive technique to bleed incubating birds without trapping: A blood-sucking bug in a hollow egg. Journal of Ornithology , 147, 115-118.

Bell CG, Lowe R, Adams PD et al. (2019) DNA methylation aging clocks: challenges and recommendations. Genome biology ,20, 1-24. 
Bichet C, Bouwhuis S, Bauch C et al. (2020) Telomere length is repeatable, shortens with age and reproductive success, and predicts remaining lifespan in a long-lived seabird. Molecular Ecology ,29 , 429-441.

Bichet C, Vedder O, Sauer-Gurth H et al. (2019) Contrasting heterozygosity-fitness correlations across life in a long-lived seabird.Molecular Ecology , 28, 671-685.

Bocklandt S, Lin W, Sehl ME et al. (2011) Epigenetic Predictor of Age. PloS one, 6 , e14821.

Bollati V, Schwartz J, Wright R et al. (2009) Decline in genomic DNA methylation through aging in a cohort of elderly subjects. Mechanisms of Ageing and Development , 130 , 234-239.

Boonekamp JJ, Salomons M, Bouwhuis S, Dijkstra C, Verhulst S (2014) Reproductive effort accelerates actuarial senescence in wild birds: an experimental study. Ecology letters , 17 , 599-605.

Bouwhuis S, Vedder O, Becker PH (2015) Sex-specific pathways of parental age effects on offspring lifetime reproductive success in a long-lived seabird. Evolution; international journal of organic evolution ,69 , $1760-1771$.

Bouwhuis S, Verhulst S, Bauch C, Vedder O (2018) Reduced telomere length in offspring of old fathers in a long-lived seabird. Biology letters, 14 .

Brooks ME, Kristensen K, van Benthem KJ, Magnusson A, Bolker BM (2017) glmmTMB Balances Speed and Flexibility Among Packages for Zero-inflated Generalized Linear Mixed Modeling. The $R$ journal , 9 , $378-400$.

Ciccarone F, Tagliatesta S, Caiafa P, Zampieri M (2018) DNA methylation dynamics in aging: how far are we from understanding the mechanisms? Mechanisms of Ageing and Development , 174, 3-17.

Day K, Waite LL, Thalacker-Mercer A et al. (2013) Differential DNA methylation with age displays both common and dynamic features across human tissues that are influenced by CpG landscape. Genome biology , 14, 1-19.

De Paoli Iseppi R, Deagle BE, Polanowski AM et al. (2019) Age estimation in a long-lived seabird (Ardenna tenuirostris) using DNA methylation-based biomarkers. Molecular Ecology Resources ,19 , 411-425.

Descamps S, Gaillard JM, Hamel S, Yoccoz NG (2016) When relative allocation depends on total resource acquisition: implication for the analysis of trade-offs. Journal of Evolutionary Biology ,29 , 1860-1866.

Erikstad KE, Fauchald P, Tveraa T, Steen H (1998) On the cost of reproduction in long-lived birds: The influence of environmental

variability. Ecology, $\mathbf{7 9}, 1781-1788$.

Ewels P, Magnusson M, Lundin S, Kaller M (2016) MultiQC: summarize analysis results for multiple tools and samples in a single report.Bioinformatics (Oxford, England) , 32, 3047-3048.

Fisher RA (1930) The genetical theory of natural selection. Oxford University Press.

Forslund P, Part T (1995) Age and Reproduction in Birds - Hypotheses and Tests. Trends in Ecology $\mathcal{G}^{3}$ Evolution, $10,374-378$.

Fraga MF, Ballestar E, Paz MF et al. (2005) Epigenetic differences arise during the lifetime of monozygotic twins.Proceedings of the National Academy of Sciences , 102, 10604-10609.

Gaillard JM, Garratt M, Lemaitre JF (2017) Senescence in Mammalian Life History Traits. In: The Evolution of Senescence in the Tree of Life (eds Shefferson R, Jones O, Salguero-Gomez R), pp. 126-155. Cambridge University Press, Cambridge.

Gu H, Smith ZD, Bock C et al. (2011) Preparation of reduced representation bisulfite sequencing libraries for genome-scale DNA methylation profiling. Nature Protocols , 6 , 468-481. 
Hamilton WD (1966) The moulding of senescence by natural selection.Journal of Theoretical Biology , 12 , $12-45$.

Hannum G, Guinney J, Zhao L et al. (2013) Genome-wide methylation profiles reveal quantitative views of human aging rates. Molecular Cell , 49 , 359-367.

Heyn H, Li N, Ferreira HJ et al. (2012) Distinct DNA methylomes of newborns and centenarians. Proceedings of the National Academy of Sciences of the United States of America , 109 , 10522-10527.

Holliday R (2006) Epigenetics: a historical overview.Epigenetics , 1 , 76-80.

Horvath S, Gurven M, Levine ME et al. (2016) An epigenetic clock analysis of race/ethnicity, sex, and coronary heart disease. Genome biology , 17, 1-23.

Horvath S, Zoller JA, Haghani A et al. (2021) Epigenetic clock and methylation studies in the rhesus macaque. GeroScience ,43, 2441-2453.

Howe K, Chow W, Collins J et al. (2021) Significantly improving the quality of genome assemblies through curation. GigaScience, 10, 1-9.

Husby A (2020) On the Use of Blood Samples for Measuring DNA Methylation in Ecological Epigenetic Studies. Integrative and Comparative Biology , 60 , 1558-1566.

Jaenisch R, Bird A (2003) Epigenetic regulation of gene expression: how the genome integrates intrinsic and environmental signals. Nature Genetics , 33, 245-254.

Kim J, Lee C, Ko BJ et al. (2021) False gene and chromosome losses affected by assembly and sequence errors. bioRxiv , 2021.04.09.438906.

Kirkwood TB (1977) Evolution of ageing. Nature, 270 , 301-304.

Klughammer J, Datlinger P, Printz D et al. (2015) Differential DNA Methylation Analysis without a Reference Genome. Cell reports , 13 , 2621-2633.

Krueger F, Andrews SR (2011) Bismark: a flexible aligner and methylation caller for Bisulfite-Seq applications. Bioinformatics (Oxford, England), $27,1571-1572$.

Krueger, F., James, F., Ewels, P., Afyounian, E. \& Schuster-Boeckler, B. (2021) FelixKrueger/TrimGalore: DOI via Zenodo (all versions). Zenodo. 10.5281/zenodo.5127898.

Langmead B, Salzberg SL (2012) Fast gapped-read alignment with Bowtie 2.Nature methods , 9 , 357-359.

Lea AJ, Vilgalys TP, Durst PAP, Tung J (2017) Maximizing ecological and evolutionary insight in bisulfite sequencing data sets. Nature ecology $\&$ evolution , 1 , 1074-1083.

Lemaitre JF, Rey B, Gaillard JM et al. (2021) DNA methylation as a tool to explore ageing in wild roe deer populations. Molecular Ecology Resources , 00 , 1-14.

Li Q, Li N, Hu X et al. (2011) Genome-wide mapping of DNA methylation in chicken. PloS one, 6 , e19428.

Lindstedt SL, Calder WA (1976) Body Size and Longevity in Birds. The Condor , 78 , 91-94.

Lu AT, Fei Z et al. (2021) Universal DNA methylation age across mammalian tissues. bioRxiv , 2021.01.18.426733.

Ludecke D (2018) sjPlot - Data Visualization for Statistics in Social Science. DOI via Zenodo. 10.5281/zenodo. 2400856

Ludecke D, Ben-Shachar MS, Patil I, Makowski D (2020) Extracting, Computing and Exploring the Parameters of Statistical Models using R.Journal of Open Source Software , 5 , 2445. 
Ludecke D, Ben-Shachar MS, Patil I, Waggoner P, Makowski D (2021) performance: An R Package for Assessment, Comparison and Testing of Statistical Models. Journal of Open Source Software , 6 , 3139.

Masser DR, Hadad N, Porter HL et al. (2017) Sexually divergent DNA methylation patterns with hippocampal aging. Aging Cell ,16 , 1342-1352.

Maegawa S, Hinkal G, Kim HS et al. (2010) Widespread and tissue specific age-related DNA methylation changes in mice. Genome Research , 20 , 332-340.

Medawar PB (1952) An Unsolved Problem of Biology: An Inaugural Lecture Delivered at University College, London, 6 December, 1951.

Melzer D, Pilling LC, Ferrucci L (2020) The genetics of human ageing.Nature reviews. Genetics , 21 , 88-101.

Meng H, Joyce AR, Adkins DE et al. (2010) A statistical method for excluding non-variable CpG sites in high-throughput DNA methylation profiling. BMC Bioinformatics, 11 , 227.

Miranda TB, Jones PA (2007) DNA methylation: The nuts and bolts of repression. Journal of Cellular Physiology , 213, 384-390.

Moiron M, Araya-Ajoy YG, Teplitsky C, Bouwhuis S, Charmantier A (2020) Understanding the Social Dynamics of Breeding Phenology: Indirect Genetic Effects and Assortative Mating in a Long-Distance Migrant. The American naturalist, 196 , 566-576.

Monaghan P, Charmantier A, Nussey DH, Ricklefs RE (2008) The evolutionary ecology of senescence. Functional Ecology ,22, 371-378.

Mulder RH, Neumann A, Cecil CAM et al. (2021) Epigenome-wide change and variation in DNA methylation in childhood: trajectories from birth to late adolescence. Human molecular genetics , 30 , 119-134.

Nisbet ICT, Apanius V, Friar MS (2002) Breeding Performance of Very Old Common Terns. Journal of Field Ornithology , 73, 117-124.

Nisbet I, Iles D, Kaneb A, Auk CMT, Makowski D (2020) Breeding performance of Common Terns (Sterna hirundo) does not decline among older age classes. American Zoologist , 137 , 1-17.

Nussey DH, Coulson T, Festa-Bianchet M, Gaillard JM (2008) Measuring senescence in wild animal populations: towards a longitudinal approach.Functional Ecology , 22, 393-406.

Prado NA, Brown JL, Zoller JA et al. (2021) Epigenetic clock and methylation studies in elephants. Aging Cell, 20, e13414.

R Core Team, (2021) R: A Language and Environment for Statistical Computing. https://www.Rproject.org, Vienna, Austria.

Raj K, Szladovits B, Haghani A et al. (2021) Epigenetic clock and methylation studies in cats. GeroScience

Rhie A, McCarthy SA, Fedrigo O et al. (2021) Towards complete and error-free genome assemblies of all vertebrate species (VGP, C genome, assembly, Eds,). Nature, 592, 737-746.

Robeck TR, Fei Z, Lu AT et al. (2021) Multi-species and multi-tissue methylation clocks for age estimation in toothed whales and dolphins. Communications Biology , 4, 1-11.

Sen P, Shah PP, Nativio R, Berger SL (2016) Epigenetic Mechanisms of Longevity and Aging. Cell , 166 , 822-839.

Sepers B, van den Heuvel K, Lindner M et al. (2019) Avian ecological epigenetics: pitfalls and promises. Journal of Ornithology , 160, 1183-1203. 
Shefferson RP, Jones OR, Salguero-Gomez R (2017) The Evolution of Senescence in the Tree of Life . Cambridge University Press.

Sheldon EL, Schrey AW, Hurley LL, Griffith SC (2020) Dynamic changes in DNA methylation during postnatal development in zebra finches Taeniopygia guttata exposed to different temperatures. Journal of Avian Biology , 51 , e02294.

Smith ZD, Gu H, Bock C, Gnirke A, Meissner A (2009) High-throughput bisulfite sequencing in mammalian genomes. Methods (San Diego, Calif.) , 48, 226-232.

Suzuki MM, Bird A (2008) DNA methylation landscapes: provocative insights from epigenomics. Nature reviews. Genetics, $\mathbf{9}, 465-476$.

Sziraki A, Tyshkovskiy A, Gladyshev VN (2018) Global remodeling of the mouse DNA methylome during aging and in response to calorie restriction.Aging Cell , 17, e12738.

Tan Q, Heijmans BT, Hjelmborg JVB et al. (2016) Epigenetic drift in the aging genome: a ten-year follow-up in an elderly twin cohort.International journal of epidemiology , 45, 1146-1158.

Tharakan R, Ubaida-Mohien C, Moore AZ et al. (2020) Blood DNA Methylation and Aging: A CrossSectional Analysis and Longitudinal Validation in the InCHIANTI Study. The journals of gerontology. Series A, Biological sciences and medical sciences , 75 , 2051-2055.

Thompson MJ, vonHoldt B, Horvath S, Pellegrini M (2017) An epigenetic aging clock for dogs and wolves. Aging , 9 , 1055-1068.

Unnikrishnan A, Freeman WM, Jackson J et al. (2019) The role of DNA methylation in epigenetics of aging. Pharmacology 85 Therapeutics , 195 , 172-185.

van de Pol M, Wright J (2009) A simple method for distinguishing within-versus between-subject effects using mixed models. Animal Behaviour , 77, 753-758.

van Noordwijk AJ, de Jong G (1986) Acquisition and Allocation of Resources - Their Influence on Variation in Life-History Tactics. The American naturalist , 128, 137-142.

Vaupel JW, Yashin AI (1985) Heterogeneity's ruses: some surprising effects of selection on population dynamics. The American statistician , 39, 176-185.

Vedder O, Moiron M, Bichet C et al. (2021a) Telomere length is heritable and genetically correlated with lifespan in a wild bird.Molecular Ecology, 256, 99.

Vedder O, Pen I, Bouwhuis S (2021b) How fitness consequences of early-life conditions vary with age in a long-lived seabird: A Bayesian multivariate analysis of age-specific reproductive values. The Journal of animal ecology , $90,1505-1514$.

Watson H, Salmon P, Isaksson C (2019) Dynamic changes in DNA methylation during embryonic and postnatal development of an altricial wild bird.Ecology and Evolution , 9 , 9580-9585.

Weidner CI, Lin Q, Koch CM et al. (2014) Aging of blood can be tracked by DNA methylation changes at just three CpG sites. Genome biology , 15, 1-12.

Williams GC (1957) Pleiotropy, Natural-Selection, and the Evolution of Senescence. Evolution; international journal of organic evolution, 11, 398-411.

Wilson AJ, Charmantier A, Hadfield JD (2008) Evolutionary genetics of ageing in the wild: empirical patterns and future perspectives.Functional Ecology , 22, 431-442.

Yuan T, Jiao Y, de Jong S et al. (2015) An Integrative Multi-scale Analysis of the Dynamic DNA Methylation Landscape in Aging.PLoS genetics, 11, e1004996. 
Yusipov I, Bacalini MG, Kalyakulina A et al. (2020) Age-related DNA methylation changes are sex-specific: a comprehensive assessment.Aging, 12, 24057-24080.

Zampieri M, Ciccarone F, Calabrese R et al. (2015) Reconfiguration of DNA methylation in aging. Mechanisms of Ageing and Development, 151, 60-70.

Zhang G, Li C, Li Q et al. (2014) Comparative genomics reveals insights into avian genome evolution and adaptation. Science ,346, 1311-1320.

Zhang H, Rebke M, Becker PH, Bouwhuis S (2015a) Fitness prospects: effects of age, sex and recruitment age on reproductive value in a long-lived seabird. The Journal of animal ecology , 84, 199-207.

Zhang H, Vedder O, Becker PH, Bouwhuis S (2015b) Contrasting between- and within-individual trait effects on mortality risk in a long-lived seabird. Ecology , $96,71-79$.

Zhang H, Vedder O, Becker PH, Bouwhuis S (2015c) Age-dependent trait variation: the relative contribution of within-individual change, selective appearance and disappearance in a long-lived seabird.The Journal of animal ecology, 84, 797-807.

\section{Data accessibility}

The common tern reference genome we developed (bSteHir1) is accessible in NCBI (https://www.ncbi.nlm.nih.gov/bioproject/560234), as well in Genome Ark (https://vgp.github.io/genomeark/Sterna_hirundo/). All RRBS data will be deposited into the public database at ENA https://www.ebi.ac.uk/ena. The final dataset and our R code will be uploaded to Dryad upon acceptance of the manuscript.

\section{Author Contributions}

SB collected the blood samples and life history data on the terns, ML and SB designed the study and ML organized sequencing of the samples. BSM analyzed the methylation data and performed the statistical analyses with the help of MM. The reference genome was generated as part of the Vertebrate Genome Project, coordinated by EDJ. OF coordinated the genome assembly; $\mathrm{BH}$ and JM were responsible for sample processing and generated the genome sequence data; CC, GF and MU-S for the genome assembly; WC, JW and $\mathrm{KH}$ curated the final reference genome. BSM and SB wrote the manuscript with input from ML, MM and EDJ. All authors commented on drafts of the manuscript.

\section{Tables and Figures}

Table 1. Result of a Generalised Linear Mixed Model with binomial error distribution testing whether variation in autosomal methylation level is explained by sex (males as a reference category) and the between(average age) and within-individual (delta age) components of age. Provided are parameter estimates and associated $95 \%$ credible intervals (95\% CI). Significant effects (p-value $<0.05$ and $95 \%$ CI which do not overlap with zero) are presented in bold.

\begin{tabular}{lllll}
\hline parameter & estimate & $\mathbf{9 5 \%} \mathbf{C I}$ & $\mathbf{z}$ & $\mathbf{p}$ \\
\hline fixed effects & & & & \\
intercept & -0.131 & $\mathbf{- 0 . 2 2 6}, \mathbf{- 0 . 0 3 5}$ & -2.677 & $\mathbf{0 . 0 0 7}$ \\
sex & -0.036 & $-0.100,0.029$ & -1.086 & 0.277 \\
average age & 0.000 & $-0.007,0.007$ & 0.093 & 0.926 \\
delta age & 0.001 & $-0.002,0.003$ & 0.514 & 0.607 \\
sex:delta age & -0.004 & $\mathbf{- 0 . 0 0 8 , - 0 . 0 0 1}$ & -2.399 & $\mathbf{0 . 0 1 6}$ \\
random effects & & & & \\
bird identity & 0.095 & $0.074,0.121$ & & \\
position identity & 2.739 & $2.714,2.764$ & &
\end{tabular}




\begin{tabular}{lllll}
\hline parameter & estimate & $\mathbf{9 5 \%} \mathbf{C I}$ & $\mathbf{z}$ & $\mathbf{p}$ \\
\hline observation & 1.263 & $1.26,1.265$ & & \\
\hline
\end{tabular}

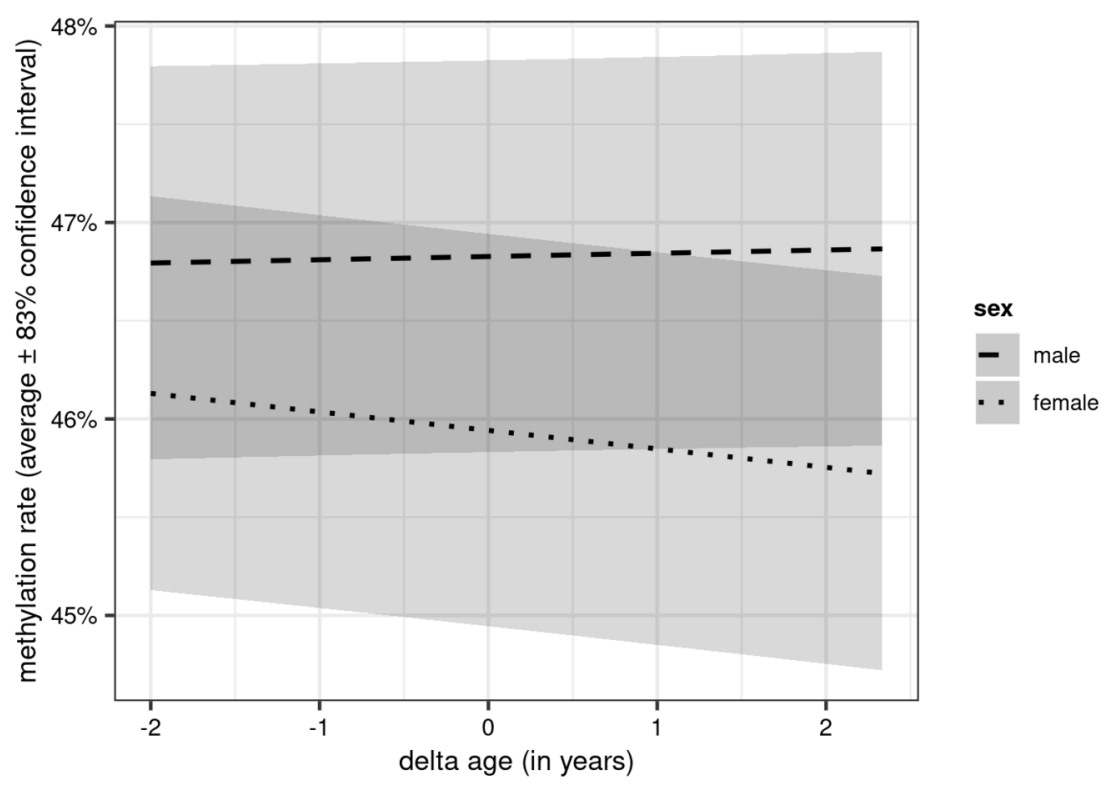

Figure 1. Autosomal DNA methylation rate declines with age within individual female, but not male, common terns. Lines represent model predictions, grey areas the $83 \%$ credible interval.

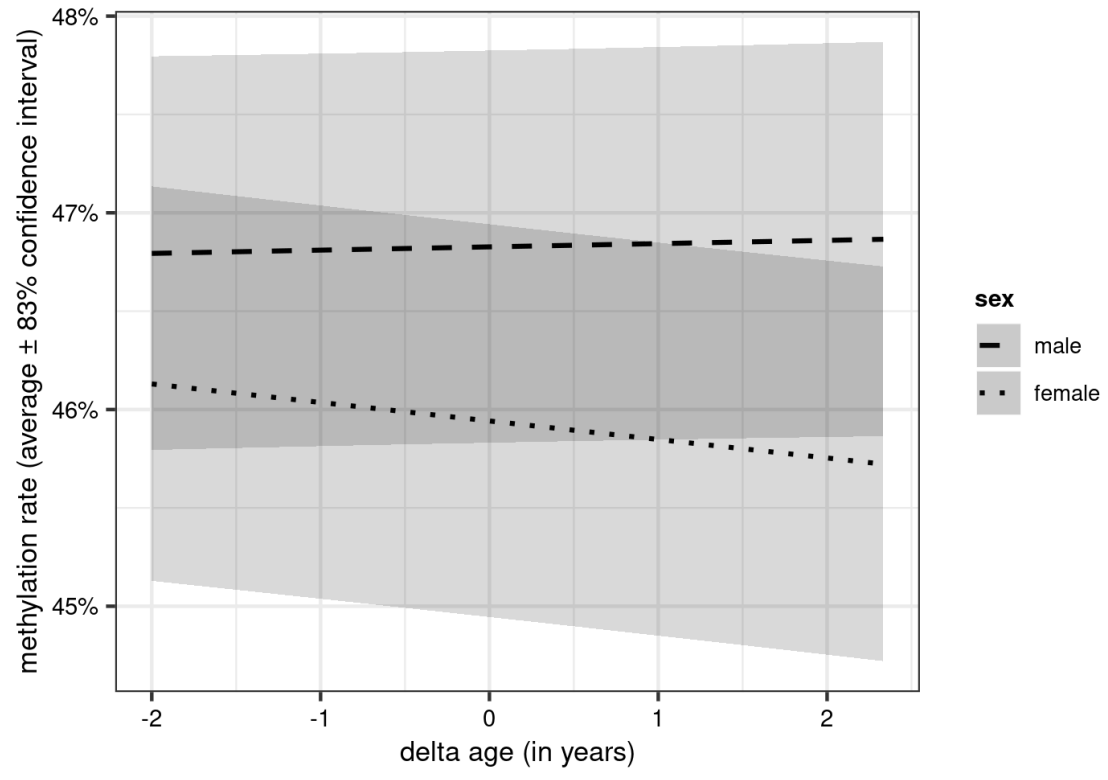




\section{Hosted file}

Table1.xlsx available at https://authorea.com/users/462472/articles/557893-sex-specificchanges-in-autosomal-methylation-rate-in-ageing-common-terns 